\section{Comparing the analgesic safety and efficacy of Diclofenac sodium tablet vs transdermal Diclofenac on postoperative third molar Extraction pain, swelling and trismus}

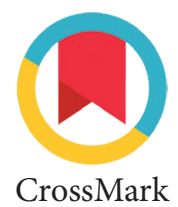

Keywords: Diclofenac sodium tablet, Impacted tooth, Transdermal diclofenac

Cite this Article: Dastagir F, Balamurugan R. 2019. Comparing the analgesic safety and efficacy of diclofenac sodium tablet vs transdermal diclofenac on postoperative third molar extraction pain, swelling and trismus. Journal of Dentomaxillofacial Science 4(2): 67-70. D0l:

Pain is one of the most common symptoms experienced by the patients after mandibular third molar surgery. Management of such pain would vary in delivering analgesics in different routes depending on the availability and intensity of drugs administered. Most prioritised class of drugs are the NSAIDS (diclofenac), which has an effective antiinflammatory, anti-pyretic and analgesic properties. When such drugs are administered in the oral route, half the dose of diclofenac reaches the systemic circulation due to first pass metabolism with potential adverse effects of gastric irritation due to high plasma concentration. ${ }^{1}$ The progress of medical science has evolved with alternate and novel routes of drug administration. One such route is transdermal route in which the drug agents are applied to the skin as a patch such that adequate amount of drug penetrates through the skin to exert

In our study we have compared and evaluated the post-operative analgesia, adverse effects, tolerability to drugs and patient compliance with the use of oral diclofenac sodium tablets and transdermal diclofenac patch following mandibular third molar surgery.

\section{Material and Methods}

This prospective, clinical, randomized control study analogue scale, swelling with three point discrimination and trismus by measuring the inter-incisal distance on 2nd and 7th postoperatively days.

Results: Patients in group II had less pain, swelling and trismus when compared with group I on 2nd and 7th postoperative days with statistical significance of $p<0.05$ respectively.

Conclusion: Transdermal diclofenac patches provide an effective way in management of postoperative pain, swelling and trismus in patients of mandibular impactions with negligible side effects and good patient compliance.

Objective: To compare the analgesic efficacy of oral diclofenac sodium to diclofenac sodium transdermal patch in the management of impacted mandibular third molars. Material and Methods: The total of 100 patients were randomly
assigned to one of the two groups. Group I patients were medicated with oral diclofenac sodium $50 \mathrm{mg}$ thrice daily for 3 days. mg placed once daily for 3 days after mandibular third molar surgery. All the patients were assessed for pain using a visual

Oral and Maxillofacial Surgeon/ former postgraduate, India
*Corresponding to: Fauziya Bastagir, Oral and Maxillofacial Surgeon/ former postgraduate, India

bala100192@gmail.com

Received: 9 January 2019 Revised: 25 February 2019 Accepted: 5 March 2019 Available online 1 August 2019

Avilable online 1 August 2019 10.15562/ jdmfs.v4i2.936

\section{Introduction} a systemic effect. which was carried out on patients who presented for surgical removal of mandibular third molars to the Department of Oral and Maxillofacial Surgery, Meenakshi Ammal Dental College, Chennai. The study sample included 100 patients (62 males, 38 females) of age ranging from 18 to 60 years with a mean age of 35 years. The study design was informed and informed consent were obtained from the patient prior to the procedure. Ethical clearance was obtained from the institutional review board. All the patients underwent surgical removal of the impacted third molar by a single surgeon.

Inclusion criteria: patients under ASA I category undergoing surgical removal of mesioangularly impacted mandibular third molars with a Pederson difficulty index ranging between 3 and 6 (Easy and Moderately difficult impactions), Patients within the age group of 18 to 60 years, non-smokers and non-diabetic patients, Adequate preoperative mouth opening of atleast $40 \mathrm{~mm}$.

Exclusion criteria: patients coming under ASA II, III and IV categories, patients having impacted mesioangular (class III), vertical, horizontal and distoangular mandibular third molars with pederson's difficulty index of above 6, history of bronchial asthma, allergic reaction to NSAIDs, patients with systemic conditions predisposing to local infections such as uncontrolled diabetes mellitus, aids or concurrent cancer chemotherapy, pa- 
tients with local factors predisposing to infections such as deep caries, periapical pathology, cyst, neoplastic lesions (or) history of radiotherapy on mandible, patients with bone pathologies like osteo arthritis and rheumatoid arthritis, known allergy to local anesthesia, aspirin, piroxicam or any other NSAID.

Randomization: the total of 100 patients were randomly assigned to one of the two groups: group I: oral Diclofenac Sodium 50mg tid was given. Group II: transdermal Diclofenac Sodium Patches 100mg placed once daily for 3 days.

Surgical procedure: the eligible patients are assigned randomly to either Group I or Group II. Intraoral preparation was done with povidone iodine solution. Anaesthesia was secured with $2 \%$ lignocaine hydrochloride with 1:80.000 adrenaline by inducing inferior alveolar nerve block, lingual nerve block and long buccal nerve block. After Subjective and objective symptoms of anesthesia was perceived, A Ward 's incision or Ward's with distal extension was placed, the mucoperiosteal flap reflected and the bone exposed. Bone removal was carried out by guttering on the buccal and the distal aspect of the tooth using a cross cut tapering fissure bur number 702. After adequate amount of bone removal the tooth was elevated and delivered from the socket. Finally the socket was irrigated with povidine iodine and saline. Sharp bony edges were smoothened with bone file and the socket was irrigated to remove bone debris. Complete haemostasis was achieved before wound closure. The wound was closed with 3-0 silk suture and the patient was given postoperative instructions. Following the procedure, postoperative medication was prescribed: group I: Cap. Amox 500mg TID. Tab. Diclofenac Sodium 50mg TID. Group II: Cap.Amox - 500mg TID. Trandermal diclofenac sodium patch placed for 3 days figure 1 and figure 2 .

Assessment: the patients were evaluated by the same independent observer postoperatively on the second and seventh postoperative days for the parameters of pain, swelling and mouth opening.

Subjective evaluation of pain: pain was evaluated using a $10 \mathrm{~cm}$ visual analogue scale (VAS), the scoring from 0 - 10 with 0 being no pain, 5 being moderate pain and 10 the worst possible pain.

Evaluation of swelling: facial swelling was determined by the craniometric method by measuring distance from the outer canthus of eye to the angle of mandible (S1), distance from tragus of ear to the corner of mouth (S2), and distance from tragus of ear to soft tissue pogonion (S3). These measurements were obtained with a string and measured in $\mathrm{mm}$ on a standard graduated scale. The average of
$\mathrm{S} 1+\mathrm{S} 2+\mathrm{S} 3$ was recorded as the amount of swelling (S).

Evaluation of trismus: maximum mouth opening was measured in mm using vernier caliper between the incisal edges of upper and lower central incisors.

\section{Results}

A total of 100 patients requiring surgical removal of impacted mandibular third molars were included in the present study, of whom 62 were men and 38 were women, with a mean average age of 35 years. The results of pain, swelling and mouth opening scores for all two groups over the 2nd and 7 th postoperative days are presented in table 1-3. The data was analysed statistically with ANOVA test and chi square Test using SPSS software.

Comparison of two groups: pain; the severity of pain was drastically reduced in transdermal patch group with a mean difference of $4.46+/-1.80$ (SD) on day 2 and $0.26+/-0.70$ (SD) on day 7 with statistical significance results of $\mathrm{p}<0.05$ respectively. Swelling; the severity of swelling was reduced in transdermal patch group with a mean difference of $14.21+/-1.08$ (SD) on day 2 and $12.81+/-0.82$ (SD) on day 7 with statistical significance results of $\mathrm{p}<0.05$ respectively. Mouth opening; the severity of mouth opening was comparatively reduced in transdermal patch group when compared with oral diclofenac group with

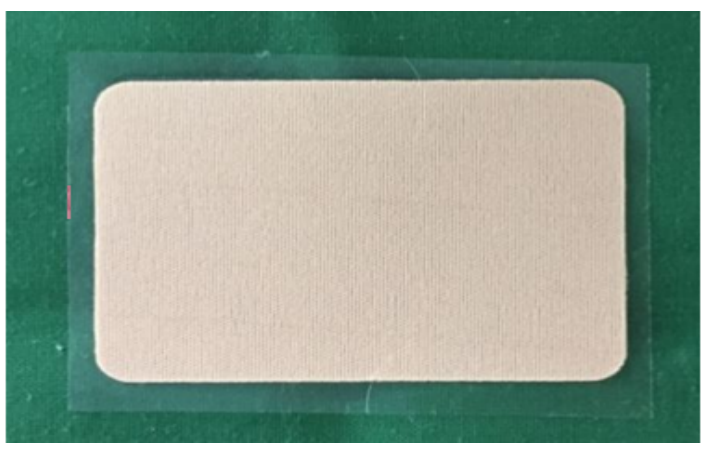

Figure 1 Diclofenac transdermal patch $100 \mathrm{mg}$

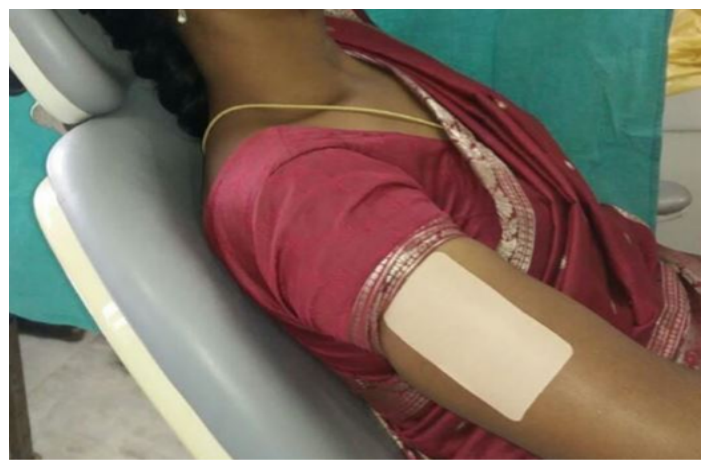

Figure 2 Transdermal patch placed in the right arm 
Table 1 Average pain scores between groups

\begin{tabular}{|c|c|c|c|c|}
\hline Days & Route & Mean & SD & P Value \\
\hline Day 2 & Oral & 6.13 & 1.80 & \\
\hline & Transderma & 4.46 & 1.74 & 0.04 \\
\hline Day 7 & $\begin{array}{l}\text { Oral } \\
\text { Transderma }\end{array}$ & $\begin{array}{l}3.86 \\
0.26\end{array}$ & $\begin{array}{l}1.95 \\
0.70\end{array}$ & 0.00 \\
\hline
\end{tabular}

Table 2 Average swelling scores between groups

\begin{tabular}{llccc}
\hline Days & Route & Mean & SD & P Value \\
\hline Day 2 & Oral & 15.41 & 1.18 & \\
& Transdermal & 14.21 & 1.08 & 0.04 \\
Day 7 & Oral & 13.60 & 1.06 & \multirow{2}{*}{0.03} \\
& Transdermal & 12.81 & 0.82 & \\
\hline
\end{tabular}

Table 3 Average mouth opening scores between groups

\begin{tabular}{llccc}
\hline Days & Route & Mean & SD & P Value \\
\hline Day 2 & Oral & 2.80 & 0.72 & \\
& Transdermal & 4.64 & 1.19 & 0.03 \\
Day 7 & Oral & 3.15 & 0.81 & \\
& Transdermal & 5.94 & 1.53 & 0.02 \\
\hline
\end{tabular}

on day 2 and $5.94+/-1.53$ (SD) on day 7 with statistical significance results of $\mathrm{p}<0.05$ respectively.

\section{Discussion}

The purpose of this study was to compare and evaluate the analgesic effect and safety of diclofenac when administered orally and as transdermal patch after mandibular third molar surgery.

NSAIDS (diclofenac) is the most widely preferred effective drug of choice with its proven advantages in minimizing pain and inflammation post injury. ${ }^{2}$ In our study two drug delivery systems were correlated, one group was oral diclofenac sodium $50 \mathrm{mg}$ and other group being transdermal diclofenac patch $100 \mathrm{mg}$ placed once daily in the right arm for 3 days. The transdermal diclofenac has usually the tendency to be subjected to absorption interferences due to the presence of anatomical barriers such as the epidermis, dermis and the underlying muscle tissue. The drug is usually retained or may undergo metabolism during its journey to the nearest vascular supply, hence, the amount of drug that reaches the circulation establishes a minimum plasma concentration. This low concentration hence leads to a lesser incidenceof systemic adverse effects; however, a larger diclofenac concentration remains lower than the other modes of administration. ${ }^{3}$

Transdermal patches offer several advantages over the oral route such as by passing first pass metabolism, slow controlled absorption, constant plasma concentration which is maintained for a longer duration, no patient dependence for drug doses, no gastric discomfort, and access flexibility of terminating the drug administration by simply removing the patch from the skin. ${ }^{2}$ As per literature all the authors reported with pain control only, after the analgesia was delivered through transdermal route post operatively while the results of our study showed a clinical and statistical significance in reduction of pain and swelling as well as better improvement in the mouth opening was also experienced by the patients in transdermal patch group when compared with oral diclofenac group. This positive outcome of our study was in similar correlation with authors ${ }^{1-4}$ in the literature.

Murthykumar et al. ${ }^{5}$ and Bachalli et al. ${ }^{6}$ compared the postoperative analgesia effect with the use of oral diclofenac and transdermal diclofenac patch. His results showed that oral diclofenac had better tolerance to pain when compared with transdermal patch on immediate postoperative day while 2 nd and 3rd post operative days had no significant correlation between the groups, while Shinde et al. ${ }^{7}$ concluded that no significant differences were elicited between transdermal patch and oral diclofenac group.

Bhargava et al. ${ }^{8}$ compared the analgesic efficacy of transdermal ketoprofen and diclofenac patch in patients who underwent atraumatic extraction of first premolars for orthodontic purpose. Through his observations his results concluded that ketoprofen patch had better analgesic efficacy when compared with diclofenac patch. His study emphasized that drugs when delivered through patch form has better efficacy and safety when compared with oral form of drugs, but the choice of analgesics delivered in transdermal route is quite challenging in the effectiveness of the drug. A comparison between transdermal patch, intramuscular injection and oral route was studied by Ural et al. ${ }^{9}$ following laparoscopic cholecystectomy and concluded that transdermal route of administering drug showed efficient analgesia as intramuscular injections and oral form without any adverse events and complications. 43 patients in transdermal patch group consumed rescue analgesia in study by Kumar et al. ${ }^{10}$ while 6 paracetamol tablets consumed by the patients in Bhasker et al. ${ }^{1}$ study and Bhargava et al. ${ }^{8}$ resulted with highest number of supportive medications in diclofenac patch group when compared with transdermal ibuprofen patch group. In our study only 6 patients had consumed supportive medications to control pain. 
On considering the drug safety 34 patients in our study presented with gastric irritation in the oral diclofenac group while no adverse effects or complications noticed in transdermal diclofenac group. Bhargava et $\mathrm{al} .{ }^{8}$ in his study reported with history of fever in 2 patients on the 1st postoperative day. He stated that it may be attributed secondary to the extraction procedure as majority of the patients tolerated the patch well with uneventful post-operative period. Shinde et al. ${ }^{7}$ reported with local irritation over the right arm in $16 \%$ of patients and $4 \%$ patients had burning sensation in abdomen in transdermal patch group.

From the observations of our study, diclofenac transdermal patch is found to be the most potent and efficacious routes of drug delivery systems in reduction of all three parameters of pain, swelling and trismus without any adverse events when compared to oral diclofenac postoperatively after mandibular third molar surgery.

\section{Conclusion}

The transdermal diclofenac patch seems to be a promising analgesic modality for the management of mild to moderate postoperative pain as well minimizes the swelling and improves the mouth opening following mandibular third molar impactions, given the evidence of its established analgesic potency with lower incidence of systemic adverse effects. Transdermal diclofenac patches provided an effective way of postoperative pain management in patients of mandibular impactions with negligible side effects and good patient compliance.

\section{Acknowledgment}

None.

\section{Conflict of Interest}

The authors declare that they have no conflict of interest.

\section{References}

1. Bhaskar H, Kapoor P, Ragini et al. Comparison of transdermal diclofenac patch with oral diclofenac as an analgesic modality following multiple premolar extraction in orthodontic patients: a cross over efficacy trial. Contemp Clin Dent 2010;1: 158-163.

2. Diwan V, Srinivasa ST, Ramreddy YK et al. A comparative evaluation of transdermal diclofenac patch with oral diclofenac sodium as an analgesic drug following periodontal flap surgery: a randomized controlled clinical study. Indian J Dent Res 2019;30: 57-60.

3. Krishnan S, Sharma P, Sharma R et al. Transdermal diclofenac patches for control of post-extraction pain. Pilot randomized controlled double-blind study. Oral Maxillofac Surg 2015;19: 5-12.

4. Dhanapal S, Sureshbabu NM. Efficacy of single dose of transdermal patch as a pre-operative analgesic in root canal treatment-a randomized clinical trial. J Pharm Sci \& Res 2016;8: 125-127.

5. Murthykumar K, Varghese S. Analgesic efficacy and safety of transdermal and oral diclofenac in postoperative pain management following periodontal flap surgery. Drug Invent Today 2019;11: 652-656.

6. Bachalli PS, Nandakumar H, Srinath N. A comparitive study of diclofenac transdermal patch against oral diclofenac for pain control following removal of mandibular impacted third molars. J Maxillofac Oral Surg 2009;8: 167-172.

7. Shinde VA, Kalikar M, Jagtap S. Efficacy and safety of oral diclofenac sustained release versus transdermal diclofenac patch in chronic musculoskeletal pain: a randomized, open label trial. J Pharmacol Pharmacother 2017;8: 166-171.

8. Bhargava D, Thomas S, Beena S. Comparison between efficacy of transdermal ketoprofen and diclofenac patch in patients undergoing therapeutic extraction-a randomized prospective split mouth study. J Oral Maxillofac Surg 2019; S0278: 30387-30388.

9. Ural UG, Yener O, Sahin H. The comparison of analgesic effects of various administration methods of diclofenac sodium, transdermal, oral and intramuscular, in early postoperative period in laparoscopic cholecystectomy operations. Pak J Med Sci 2014;30: 96-100.

10. Kumar V, Gupta S, Verma R. Evaluation of the role of transdermal diclofenac patch (nupatch) in management of pain in postperative patients. Int J Contemp Med Res 2017;4: 493-496.

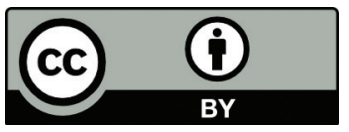

This work is licensed under a Creative Commons Attribution 